\title{
Electrodeposition of Metallic Nanowires in Nanoporous Polycarbonate Films
}

\author{
Takeshi Ohgai $^{1, a}$, Masayuki Mizumoto ${ }^{1, b}$ Shigeki Nomura² and Akio Kagawa ${ }^{1, c}$ \\ ${ }^{1}$ Department of Materials Science and Engineering, Nagasaki University, \\ ${ }^{2}$ Deparment of Materials Scinece, Graduate School of Science \& Technology, Nagasaki University, \\ 1-14 Bunkyo-machi, Nagasaki 852-8521, JAPAN \\ a ohgai@net.nagasaki-u.ac.jp, ${ }^{\mathrm{b}}$ mizumoto@net.nagasaki-u.ac.jp, ${ }^{\mathrm{c}}$ akagawa@net.nagasaki-u.ac.jp
}

Keywords: Electrodeposition, Nanowire, Nanopore, Polycarbonate, Nickel, Cobalt, Iron

\begin{abstract}
A polycarbonate membrane filter with numerous cylindrical nanopores was used as a template for growing metallic nanowires such as $\mathrm{Ni}$, Co and $\mathrm{Fe}$. The nanoporous template with pore-diameter of $150 \mathrm{~nm}$, pore-length of $6000 \mathrm{~nm}$, and pore-density of $10^{8}$ pore $\mathrm{cm}^{-2}$ was modified as a cathode with sputter-deposited gold layer. Inside the nano-pores, the metallic nanowires were electrochemically deposited from an acidic sulfate solution containing metal ions. The growth rate of metallic nanowires depended on the cathode potential during electrodeposition. The diameter of electrodeposited nanowires corresponded to that of nanopores in the template. TEM diffraction pattern suggested that each metallic nanowire composed of a single crystalline structure.
\end{abstract}

\section{Introduction}

Nano-sized metallic wires with several tens nano-meter in diameter and several micro-meter in length [1-4], have received much attention due to an increasing interest in wires close_to or below the critical size limit for quantum effects. The metallic nanowire can be applied to the optoelectronic devices in a flat panel display and the magnetic devices in a high density recording media. Metallic nanowires can be synthesized by manipulating metallic atoms using a scanning tunneling microscope and by electrochemically depositing metallic atoms into a porous template with numerous cylindrical nanopores [5-11]. Nanoporous templates such as anodized aluminum oxide films with high density of nano-pores (about $10^{8} \sim 10^{10}$ pores ${ }^{\bullet} \mathrm{cm}^{-2}$ ) have been used so far for electrodeposition of nanowires [12-17]. Template synthesis technique such as fabricating nano-pores and electrodepositing nanowires requires precise techniques controlling position of each nano-pore, inter-pore distance, pore diameter and pore shape [18-20]. Lithographic galvanoforming process using laser, electrons and ions beam could satisfied the above requirements to synthesize new nano-electronic devices. In this study, ion track-etched polycarbonate membrane filters with numerous cylindrical nano-pores was used as a template for growing ferro-magnetic metals nanowires.

\section{Experimental Procedure}

A gold thin layer with $500 \mathrm{~nm}$ in thickness was sputter-deposited on a commercially available ion track-etched polycarbonate membrane filters with nominal pore-diameter of $100 \mathrm{~nm}$, pore-length of $6000 \mathrm{~nm}$ and pore-density of $10^{8}$ pore $\mathrm{cm}^{-2}$. This membrane filter with a gold layer was used as a cathode for growing metallic nanowires. Electrolytic solution was prepared by dissolving $\mathrm{MSO}_{4} \cdot 7 \mathrm{H}_{2} \mathrm{O}\left(120 \mathrm{~g} \cdot \mathrm{L}^{-1}\right)[\mathrm{M}: \mathrm{Ni}, \mathrm{Co}, \mathrm{Fe}]$ and $\mathrm{H}_{3} \mathrm{BO}_{3}\left(45 \mathrm{~g} \cdot \mathrm{L}^{-1}\right)$ into the water purified by an ion exchange resin. To determine the optimum deposition potential for growing nanowires, cathodic polarizarion curves of the templates were investigated in each electrolytic solution. At each optimum cathode potential, the nanowires were potentio-statically deposited. To determine the nanowire growth rate, time dependence of cathodic current was monitored. After the electrodeposition, 
polycarbonate membrane filters were dissolved in organic solvent and the remains consisted of nanowires and a gold layer was served as a sample for SEM observation. To obtain a cross-sectional image of a nanowire, the electrodeposited nanowire supported in a membrane filter was thinned by ion-milling technique and the sample was served for TEM observation.

\section{Results and Discussion}

\section{Electrodeposition behavior of metals.}

SEM image of the polycarbonate membrane filter with a sputter-deposited gold layer is shown in Figure 1. The pore-diameter is estimated to be around $150 \mathrm{~nm}$, which is longer than the nominal pore-diameter of $100 \mathrm{~nm}$. This reason could be caused by the thermal expansion of polycarbonate membrane at the fabrication process of sputter-depositing gold layer on it.

Cathodic polarization curve of the polycarbonate membrane filter with gold layer is shown in Figure 2. The measurement was conducted in the solution containing $\mathrm{Ni}^{2+}$ ions. According to the concentration of $\mathrm{Ni}^{2+}$ ions in the solution, the equilibrium potential of $\mathrm{Ni}$ is estimated to be around $-0.46 \mathrm{~V}$ (vs. $\mathrm{Ag} / \mathrm{AgCl}$ ). The cathodic current occurs at around $-0.2 \mathrm{~V}$, which is more-noble than the equilibrium potential of Ni. This current is mainly caused by the reduction of hydrogen ions. With increasing the cathodic current upto $10^{-5} \mathrm{~A}$, the cathode potential polarizes to be around $-0.7 \mathrm{~V}$. This polarization could be caused by the diffusion limit of $\mathrm{H}^{+}$ions from the bulk solution to the surface of cathode. However, at the potential less-than $-0.7 \mathrm{~V}$, the cathodic current increases again. Usually, electrodeposition of $\mathrm{Ni}, \mathrm{Co}$ and $\mathrm{Fe}$ from aqueous solution accompanies with a significant overpotential [21-23]. Therefore, this increase in cathodic current is mainly due to an increase in reduction current of $\mathrm{Ni}$. Furthermore, with increasing cathodic current upto $10^{-2} \mathrm{~A}$, the cathode potential polarizes significantly to be less-noble region rahter than $-1.5 \mathrm{~V}$. This huge polarization could be caused by the diffusion limit of $\mathrm{Ni}^{2+}$ ions. According to this electrodeposiotion behavior of $\mathrm{Ni}$, the optimum deposition potential is estimated to be around -0.8 to $-1.2 \mathrm{~V}$. Electrodeposition behavior of $\mathrm{Co}$ and $\mathrm{Fe}$ also showed similar tendency as well as the case of Ni.

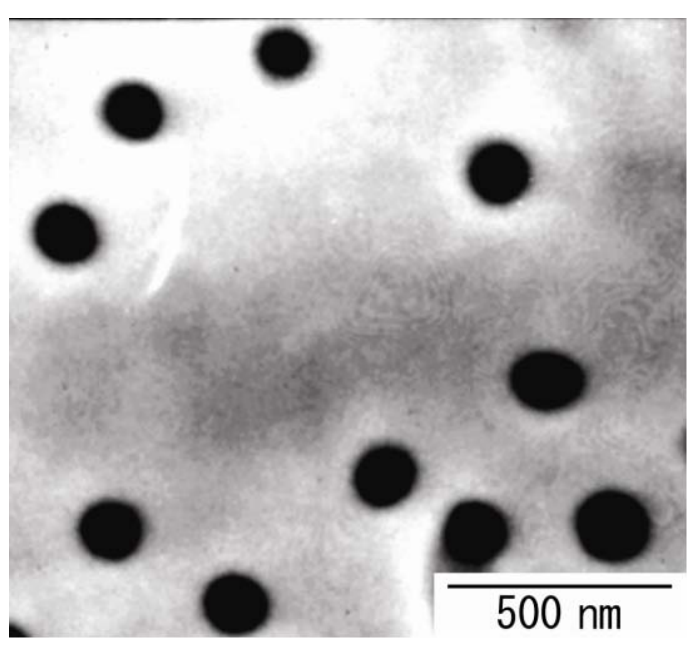

Fig.1 SEM image of the polycarbonate membrane filter with a sputter-deposited gold layer.

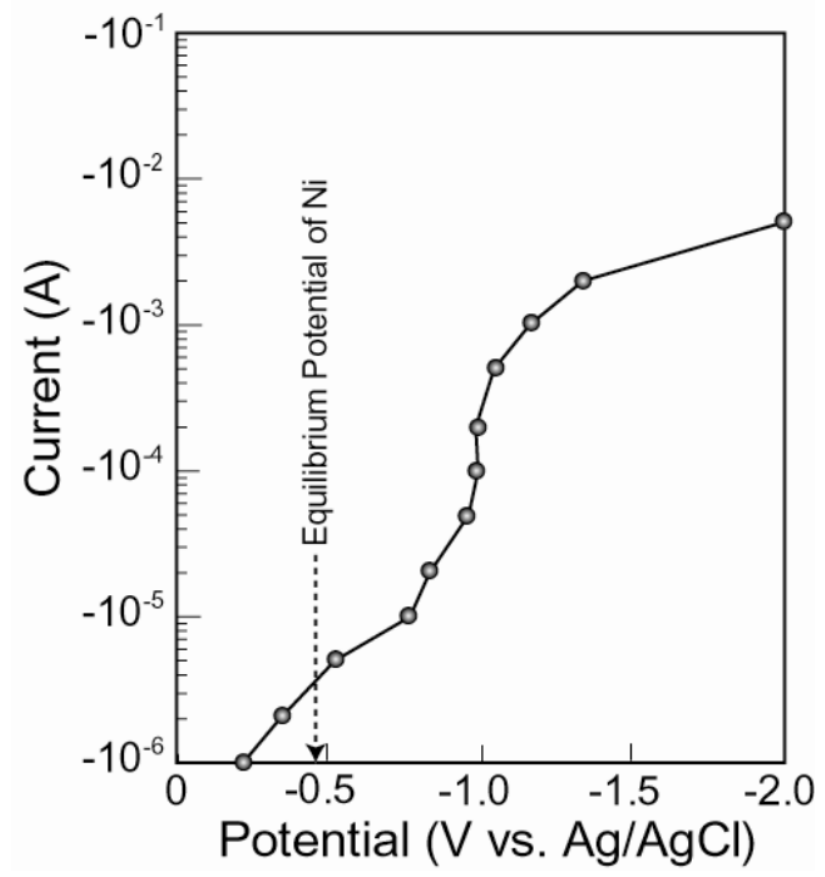

Fig.2 A polarization curve measured during $\mathrm{Ni}$ electrodeposition from an aqueous solution containing $\mathrm{Ni}^{2+}$ ions. 
Nanowire growth. Effect of cathode potential on the time-dependence of cathodic current is shown in Figure 3. During the electrodepostion of $\mathrm{Ni}$, cathode potential was fixed to $-0.8,-0.9,-1.0,-1.1$ and $-1.2 \mathrm{~V}$. When the potential was fixed to $-1.0 \mathrm{~V}$, the cathodic current reached upto $1 \mathrm{~mA}$ within several tens minutes. Then, the current rapidly decresed to $0.4 \mathrm{~mA}$ and kept the value upto $1000 \mathrm{sec}$. At this stage, reduction of Ni occurs inside the nano-pores. If the electrodeposition of metal proceeds inside of nano-pores, the reduction current value is effected by the concentration of metal ions in the nano-pores. With increasing electrodeposition time, the concentration of metal ions in the nano-pores will decrease because metal ions are consumed by electrodeposition process. On the other hand, the metal ions will diffuse from the bulk solution to the inside of nano-pores. At the final stage, the cathodic current quickly increases at around $1000 \mathrm{sec}$. Here, the nanowires are grown at surface of the template and huge bulk Ni deposits could be formed. At the cathode potential of $-1.0 \mathrm{~V}$, growth rate of Ni nanowires can be estimated as around $6 \mathrm{~nm} \cdot \mathrm{sec}^{-1}$. The growth rate of nanowires increases with increase of cathode potential. Growth rates of $\mathrm{Co}$ and Fe nanowires were also estimated as around 10 and $6 \mathrm{~nm} \bullet \mathrm{sec}^{-1}$ at $-1.0 \mathrm{~V}$. The over potential for hydrogen evolution on $\mathrm{Ni}$ are smaller than that on Co [24]. Hence, current efficiency for Co deposition could be higher than that of $\mathrm{Ni}$.

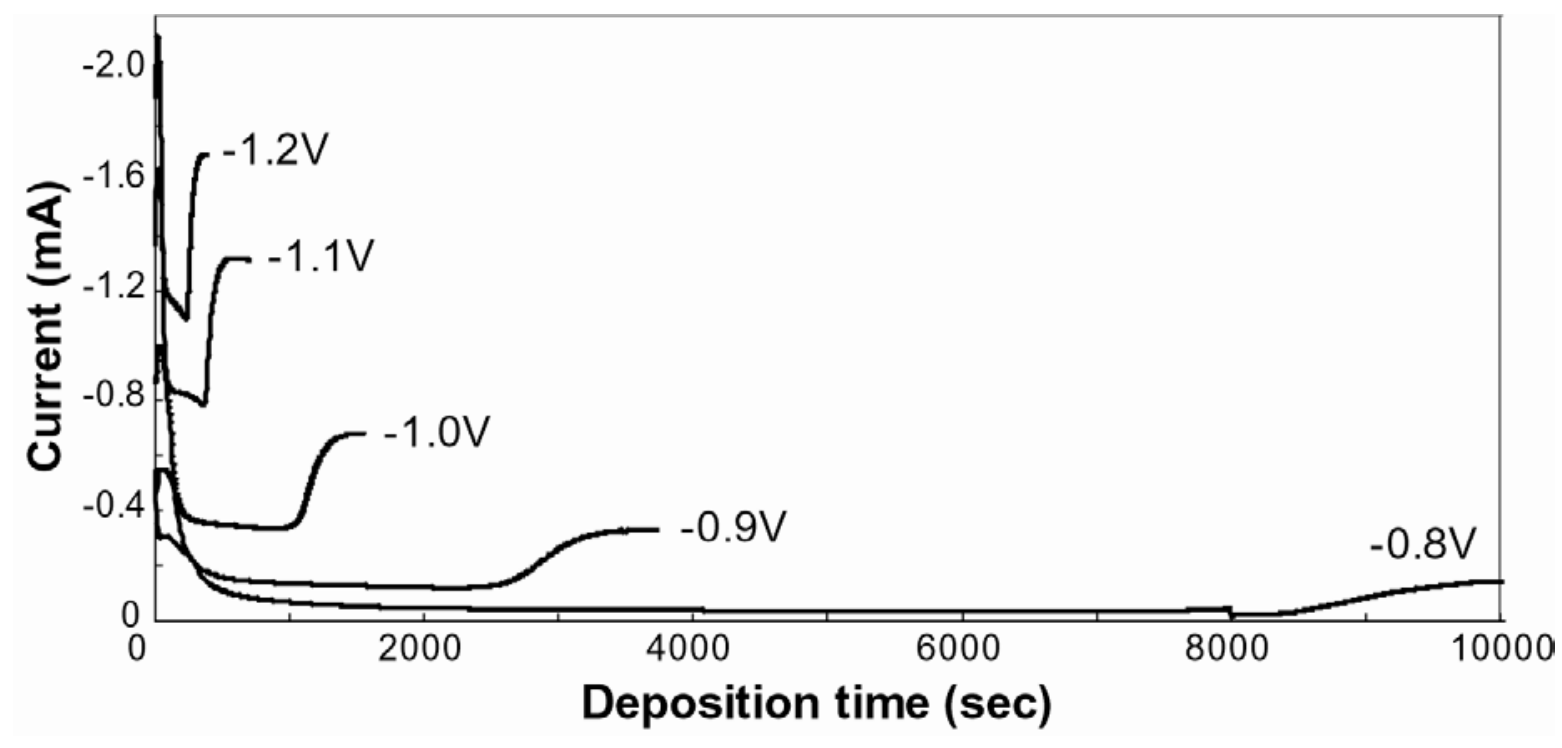

Fig. 3 Effect of cathode potential on the time-dependence of cathodic current during electrodeposition of Ni nanowires.

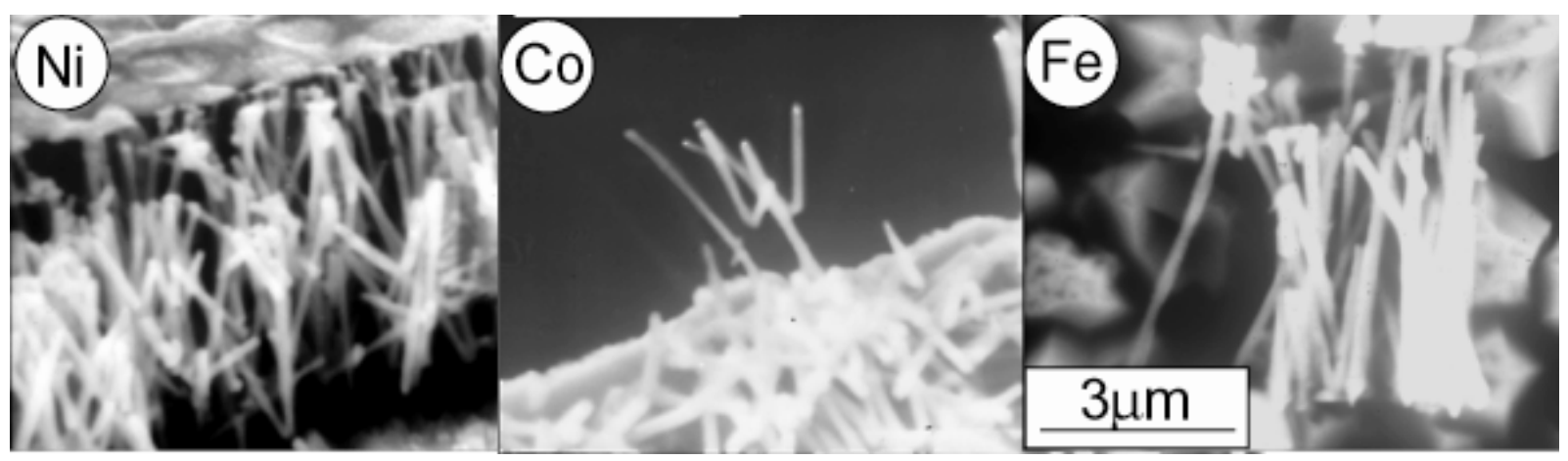

Fig. 4 SEM images of electrodeposited Ni, Co and Fe nanowires separated from polycarbonate membrane filters. 
Structure of nanowires. SEM images of $\mathrm{Ni}$, Co and Fe nanowires separated from the polycarbonate templates are shown in Figure 4. Cylindrical shape of nanowires was almost transferred from that of nano-pores. Aspect ratio of the nanowires reaches upto 40. The length of nanowire can be ranged by controlling deposition time upto the length of nanowire. On the other hand, the diameter of nanowire is fixed to the size of nanopore. TEM bright images and diffraction patterns of electrodeposited Ni, Co and Fe nanowires supported in a template are shown in Figure 5. Diameter of the nanowire can be estimated to be around $150 \mathrm{~nm}$, which corresponds well to the diameter of nanopores determined from SEM images as shown in Figure 1. On the other hand, each diffraction pattern is composed of spots, which means each sample consists of a single crystal. Electrodeposits of iron-group metals are usually composed of poly-crystals with diameter of several hundreds $\mathrm{nm}$ [25]. To obtain nano-crystalline or amorphous state deposits, huge over potential is necessary to create numerous nucleuses during electrodeposition. In this study, pure metal electrodeposits inside of nanopores without any significant overpotential. Therefore, without any crystalline seeds, single crystalline nanowires can be easily grown. TEM diffraction pattern of each nanowire also determined that the crystal direction of the long-axis as Ni [111], Co [001] and Fe [210].

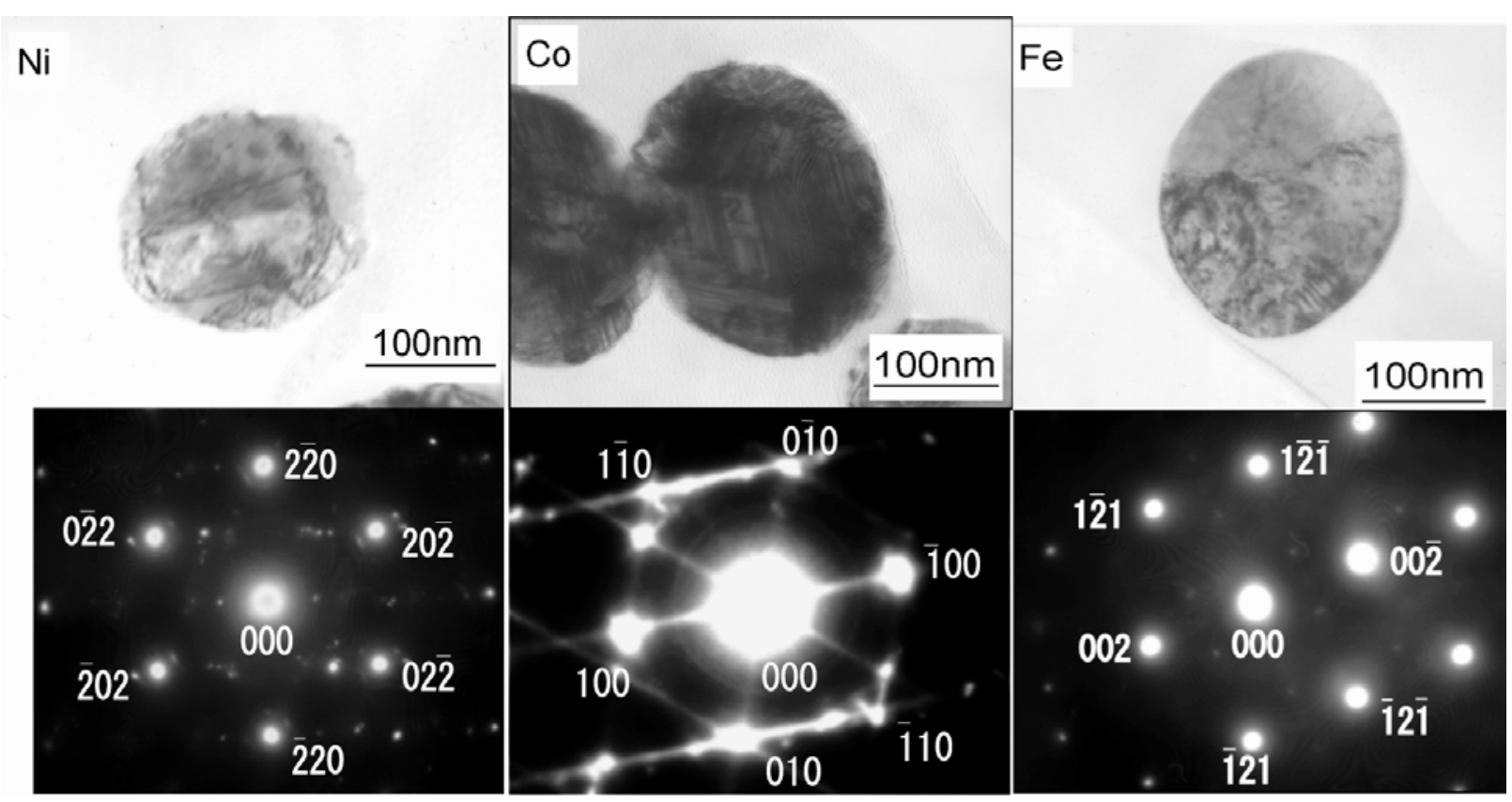

Fig. 5 TEM bright images and diffraction patterns of electrodeposited Ni (a), Co (b) and Fe (c) nanowires supported in menbrane filters.

\section{Conclusions}

The optimum deposition potential region for growing Ni, Co and Fe nanowires is determined to be around -0.8 to $-1.2 \mathrm{~V}$, which is more-noble than the diffusion limit of $\mathrm{Ni}^{2+}$ ions. The growth rate of nanowires increased with increase of cathode potential and the growth rate of $\mathrm{Ni}$ nanowires was around $6 \mathrm{~nm} \cdot \mathrm{sec}^{-1}$ at the cathode potential of $-1.0 \mathrm{~V}$, which is quite faster than that of sputter-deposited metal. The shape of electrodeposited nanowires corresponds well that of nanopores and the aspect ratio reached up to 40 . The each nanowire could be composed of single crystal. 


\section{Acknowledgements}

This work was supported in part by Izumi Science \& Technology Foundation (H16-J-139), Saneyoshi Scholarship Foundation (No.1708), Mitutoyo Association for Science \& Technology (MAST), and Japan Society for the Promotion of Science, Grant-in-aid for Young Scientists (B) (No.17760581).

\section{References}

[1] C. R. Martin, Adv. Mater. 3, 457 (1991).

[2] T. M. Whitney, J. S. Jiang, P. C. Searson, and C. L. Chien, Science 261, 1316 (1993).

[3] I. Chlebny, B. Doudin, and J.-Ph. Ansermet, Nanostruct. Mater. 2, 637 (1993).

[4] C. R. Martin, Science 266, 1961 (1994).

[5] L. Piraux, J. M. George, J. F. Despres, C. Leroy, E. Ferain, R. Legras, K. Ounadjela, A. Fert, Appl. Phys. Lett. 65, 2484 (1994).

[6] A. Blondel, J. P. Meier, B. Doudin, and J.-Ph. Ansermet, Appl. Phys. Lett. 65, 3019 (1994).

[7] K. Liu, K. Nagodawithana, P. C. Searson, and C. L. Chien, Phys. Rev. B 51, 7381 (1995).

[8] R. Ferre, K. Ounadjela, J. M. George, L. Piraux, S. Dubois, Phys. Rev. B. 56, 14066 (1997).

[9] G. P. Heydon, S. R. Hoon, A. N. Farley, S. L. Tomlinson, M. S. Valera, K. Attenborough and W. Schwarzacher, J. Phys. D: Appl. Phys. 30, 1083 (1997).

[10] J. E. Wegrowe, D. Kelly, A. Franck, S. E. Gilbert, and J.-Ph. Ansermet, Phys. Rev. Lett. 82, 3681 (1999).

[11] P. R. Evans, G. Yi, W. Schwarzacher, Appl. Phys. Lett. 76, 481 (2000).

[12] D. Aimawlawi, N. Coombs, and M. Moskovits, J. Appl. Phys. 70, 4421 (1991).

[13] A. Tayaoka, E. Tayaoka, and J. Yamasaki, J. Appl. Phys. 79, 6016 (1996).

[14] P. Forrer, F. Schlottig, H. Siegenthaler, and M. Textor, J. Appl. Electrochem. 30, 533 (2000).

[15] K. Nielsch, F. Müller, A. P. Li, and U. Gösele, Adv. Mater. 12, 582 (2000).

[16] A. J. Yin, J. Li, W. Jian, A. J. Bennett, and J. M. Xu, Appl. Phys. Lett. 79, 1039 (2001).

[17] G. Sauer, G. Brehm, S. Schneider, K. Nielsch, R. B. Wehrspohn, J. Choi, H. Hofmeister, and U. Gösele, J. Appl. Phys. 91, 3243 (2002).

[18] T. Ohgai, X. Hoffer, L. Gravier, J. E. Wegrowe , J.-Ph. Ansermet, Nanotechnology 14, 978 (2003).

[19] T. Ohgai, X. Hoffer, A. Fabian, L. Gravier, J.-Ph. Ansermet, J. Mater. Chem. 13, 2530 (2003).

[20] T. Ohgai, L. Gravier, X. Hoffer, M. Lindeberg, K. Hjort, R. Spohr, J.-Ph. Ansermet, J. Phys. D: Appl. Phys. 36, 3109 (2003).

[21] K.E. Heusler, Zeit. Electrochimie 62, 582 (1958)

[22] J. O’M. Bockris and H. Kita, J. Electrochem. Soc. 108, 676 (1961).

[23] K. Higashi, H. Fukushima, T. Urakawa, T. Adaniya, K. Matsudo, J. Electrochem. Soc. 128, 2081 (1981).

[24] H. Nakano, T. Ohgai, H. Fukushima, T. Akiyama, R. Kammel, METALL, 55, 676 (2001).

[25] L. T. Romankiw and D. A. Thompson, Properties of Electrodeposits: Their Measurement and Significance (Edited by R. Sard, H. Leidheiser and F. Ogburn, The Electrochemical Society), 389 (1975). 